\title{
A Randomized Controlled Study of the Yi Qi Gu Biao Pill in the Treatment of Frequent Exacerbator Phenotype in Chronic Obstructive Pulmonary Disease (Lung and Spleen Qi Deficiency Syndrome)
}

\author{
Gao Zhen, Jing Jing, Xu Dan, Li Zheng, Li Fengsen, and Sun Qi \\ National Clinical Research Base of Traditional Chinese Medicine, Traditional Chinese Medicine Hospital Affiliated to Xinjiang Medical \\ University, Urumqi 830000, China \\ Correspondence should be addressed to Li Fengsen; fengsen602@163.com
}

Received 3 July 2017; Revised 7 October 2017; Accepted 29 October 2017; Published 4 December 2017

Academic Editor: Albert S. Yeung

Copyright (C) 2017 Gao Zhen et al. This is an open access article distributed under the Creative Commons Attribution License, which permits unrestricted use, distribution, and reproduction in any medium, provided the original work is properly cited.

\begin{abstract}
Objective. To evaluate the efficacy and safety of Yi Qi Gu Biao (YQGB) pill in treating frequent exacerbator phenotype in chronic obstructive pulmonary disease (lung and spleen qi deficiency syndrome) (FEPCOPD). Methods. This prospective, randomized, double-blind, controlled study assessed 112 cases (64 included) of FEPCOPD treated at the outpatient department in our hospital in January-August 2016. The patients were randomly divided into YQGB and placebo $(\mathrm{Pb})$ and treated for three months. Lung function, CAT, mMRC, and TCM symptom scores (TCMs) were observed. Results. Compared with Pb, YQGB showed decreased wheezing symptom scores (WSs) and TCMs at one month and decreased CAT and TCMs at three months. From one to three months, CAT, cough, sputum, WSs, and TCMs in YQGB were lower than pretreatment values. But in Pb, CAT was lower than pretreatment values after one month; CAT, sputum, and TCMs were lower than pretreatment values after two months; CAT, cough, sputum, WSs, and TCMs were lower than pretreatment values after three months. Conclusion. Yi Qi Gu Biao pill can improve wheezing, health status, and TCMs in FEPCOPD and also can shorten the durations of cough, sputum, and wheezing. This trial is registered in the Clinical Trials Registry of China: ChiCTR-IOR-15007542 (on 8 December 2015).
\end{abstract}

\section{Introduction}

The clinical phenotype refers to disease characteristics associated with clinical manifestations such as symptoms, acute exacerbation, treatment response, disease progression, and death that can reflect the differences among COPD patients [1] and has constituted a hot research topic for chronic obstructive pulmonary disease (COPD) in recent years. Phenotypic identification not only helps understand the differences between COPD types, but also more importantly has potential diagnostic and therapeutic significance; in addition, it can better reflect the heterogeneity of COPD, prompting a more comprehensive and in-depth research in COPD. According to the relationship between the clinical course of COPD and prognosis, as well as the different responses of currently available treatment methods, three phenotypes can be distinguished, including frequent exacerbator phenotype,
COPD-asthma overlap, and emphysema-airway hyperresponsiveness [2]. The frequent exacerbator phenotype is the most frequently encountered in COPD patients; it occurs not only in the severe phase of COPD, but also in $22 \%$ of patients with GOLD grade 2 disease [3]. The frequent exacerbator phenotype in COPD refers to patients with more than two yearly disease episodes, with oral administration of glucocorticoids and/or antibiotics needed each time, or those requiring hospitalization [1]. The pathophysiology underlying the frequent exacerbator phenotype is complex, with increased airway and systemic inflammation, dynamic lung hyperinflation, changes in the bacterial colonization lower airways, and a possible increased susceptibility to viral infections. Patients with the frequent exacerbator phenotype are also at increased risk of comorbid extrapulmonary diseases, including cardiovascular disease, gastroesophageal 
reflux, depression, osteoporosis, and cognitive impairment. Overall, these patients have poorer health status, accelerated loss of forced expiratory volume over 1s (FEV1), worsened quality of life, and increased hospital admissions and mortality, contributing to increased exacerbation susceptibility and perpetuation of the frequent exacerbator phenotype [4]. Therefore, the frequent exacerbator phenotype in COPD is well worthy of special attention from clinicians and scientists.

A previous research demonstrated that [5] Yi Qi Gu Biao pill can prolong the interval of acute exacerbation in COPD patients by 77.68 days, while reducing the number of acute exacerbations by $1.42 / 6$ months. Moreover, its therapeutic effects are consistent with the main treatment purpose for the frequent exacerbator phenotype in COPD, that is, reduction of the number of acute exacerbations. Therefore, this randomized controlled study assessed the efficacy and safety of Yi Qi Gu Biao pill in treating the frequent exacerbator phenotype in COPD (lung and spleen qi deficiency syndrome) [6].

\section{Materials and Methods}

2.1. Diagnostic Criteria. The diagnostic criteria of COPD were based on "the Global Strategy for Diagnosis, Treatment, and Prevention of Chronic Obstructive Pulmonary Diseases (Revised Edition in 2011)" [7]. The diagnostic criteria for the frequent exacerbator phenotype in COPD were $[1,3]$ more than two yearly disease onset events, requiring oral administration of glucocorticoids and/or antibiotics and requirement for hospitalization during treatment. Three years were used as a prospective endpoint.

2.2. TCM Diagnosis and Criteria of Syndrome Differential Classification. Diagnostic criteria for lung and spleen qi deficiency syndrome were based on the "diagnostic criteria of TCM syndromes for chronic obstructive pulmonary disease (2011 revision)" proposed by the Committee of Chinese Medicine Association of Internal Medicine Branch of the Pulmonary Disease [8].

2.3. Inclusion Criteria. The following inclusion criteria were adopted: (1) diagnostic criteria for chronic obstructive pulmonary disease at a stable stage; (2) diagnostic criteria of TCM syndrome of qi deficiency of lung and spleen; (3) age between 40 and 75 years; (4) survival period greater than three months; (5) good compliance, agreement to cooperate in this study, and signed informed consent.

2.4. Exclusion Criteria. Patients were excluded due to the following: (1) comorbidity with diseases such as bronchial asthma, tuberculosis, bronchiectasis, pulmonary cystic fibrosis, lung cancer, lung abscess, and congestive heart failure; (2) combined primary diseases such as severe cardiovascular and cerebrovascular diseases, liver and kidney ailments, and hematopoietic system diseases; (3) pregnant or lactating women; (4) mental diseases; (5) disability such as blindness, deafness, mental retardation, and physical impairment; (6) allergy or contraindications to the experimental drugs; (7) combined with tumors; (8) congenital or acquired immunodeficiency; and (9) implication in other clinical experiments within the past month.

2.5. Withdrawal Criteria. The following were considered: (1) patient change of medical prescription half-way or supplementation of a combination of nonprescribed drugs, especially those that have large impact on the experimental drugs, affecting judgment of effectiveness and safety; (2) development of allergic reactions or serious adverse events; (3) other complications and special physiological changes disqualifying from further testing; and (4) voluntary withdrawal.

2.6. Administration Method [5]. The YQGB group was treated with the drug during stable stage as recommended by "the Global Strategy for the Diagnosis, Treatment, and Prevention of Chronic Obstructive Pulmonary Disease (2011 revision)." Meanwhile, oral administration of the Yi Qi Gu Biao pill (10 pills/time and $0.19 \mathrm{~g} /$ pill) was performed three times every day with a total treatment course of 12 weeks. The $\mathrm{Pb}$ group was treated based on the "the Global Strategy for the Diagnosis, Treatment, and Prevention of Chronic Obstructive Pulmonary Disease (the 2011 revision)." Meanwhile, oral administration of the placebo (10 pills/time and $0.19 \mathrm{~g} /$ pill) was performed three times every day with a total treatment course of 12 weeks.

\subsection{Observation Outcomes and Methods}

2.7.1. Pulmonary Function Test. The pulmonary function of patients was tested on a HI-101 pulmonary function instrument manufactured by CHEST Co., Ltd., Japan. Before the test, the investigators first explained the specific operational procedures and requirements to the subjects to ensure accurate and reliable results. All operations were completed by the same technician, and the following pulmonary function indicators were detected: forced expiratory volume in one second (FEV1) and FEV1 percentage in the predicted value (FEV1\% pred), forced vital capacity (FVC) and FVC percentage in the predicted value (FVC\% pred), and FEV1/FVC.

\subsubsection{Assessment Methods and Standards of COPD Assessment} Test (CAT). The Chinese version of the CAT scoring questionnaire was used [9]. The CAT questionnaire comprises a total of eight questions, including cough, sputum, chest tightness, the feeling of climbing or climbing a staircase, housework, degree of confidence away from home, sleep, and energy. The score for each question from mild to severe disease was $0-5$ points; the total score was calculated after scoring each question by the patients, and CAT scores ranged between 0 and 40 points. Upon definite diagnosis, the same researcher explained the questionnaire contents and the scoring method to the patients. The patients filled the questionnaire independently without reminder. Finally, two investigators evaluated the scores.

2.7.3. Classification Criteria of Modified Medical British Research Council (mMRC) [10]. The disease was graded 
as follows: 0, dyspnea occurring only during exercise; 1, shortness of breath only after fast walking on a flat surface or walking up a small slope; 2 , due to shortness of breath, slower walking on a flat surface than the peers or need to stop for rest; 3 , stop breathing after walking for about 100 meters on a flat surface or a few minutes; 4 , inability to leave home due to severe dyspnea, or dyspnea occurring during dressing and undressing.

2.7.4. TCM Syndrome Score. The TCM syndrome score was based on the quantitative criteria of chronic bronchitis symptom standards in "Guidelines for Clinical Research of Chinese Drugs," including the eight syndromes of cough, sputum, wheezing, weight loss, fatigue, poor appetite, abdominal distension, and loose stool.

2.8. Statistical Methods. Group-wise comparison between the YQGB and $\mathrm{Pb}$ groups was performed by independent samples $t$-test; comparison before and after the treatment for each group was assessed by paired $t$-test. Chi-square test was used for categorical variables. For the CAT, the minimally important difference is generally accepted as a 2point change, so chi-square test was used for categorical data $\geq 2$ points and $<2$ points. A $P$ value of $<0.05$ was deemed statistically significant. All data were analyzed with SPSS version 17.0 (IBM Corp., Armonk, NY, USA).

\section{Results}

3.1. General Data. The 112 subjects were follow-up visit patients at the National Clinical Research Base of Traditional Chinese Medicine of the Affiliated TCM Hospital of Xinjiang Medical University, between January 2016 and August 2016. A randomized, double-bind, single-treatment, and parallel controlled experimental design was used. The patients were divided into the YQGB and $\mathrm{Pb}$ groups, with each group involving 56 patients. After exclusion, there were 33 patients in the YQGB group and 31 in the $\mathrm{Pb}$ group. There were no statistically significant differences in gender, height, weight, age, BMI, pulmonary function, CAT, mMRC, and TCM syndrome score (Table 1) between the two groups of patients.

\subsection{Group-Wise Comparison}

3.2.1. Pulmonary Functions between YQGB and Pb Groups. There were no statistical significant differences in pulmonary functions between the YQGB and $\mathrm{Pb}$ groups, within the three months of treatment (Figure 1).

3.2.2. CAT, $m M R C$, and TCM Syndrome Scores. Between the YQGB and $\mathrm{Pb}$ groups, the YQGB group showed a reduced score of wheezing symptom at one month compared with the $\mathrm{Pb}$ group $(P<0.01)$; TCM syndrome score was reduced as well $(P<0.01)$. The YQGB group showed decreased CAT $(P<0.05)$ and TCM syndrome $(P<0.05)$ scores at three months compared with the $\mathrm{Pb}$ group (Figure 2, Table 2).
TABLE 1: Characteristics of the study groups.

\begin{tabular}{lcc}
\hline & YQGB & $\mathrm{Pb}$ \\
\hline Demographic data & $n=33$ & $n=31$ \\
Age (years) & $68.73 \pm 7.16$ & $70.81 \pm 5.50$ \\
Gender (male/female) & $26 / 7$ & $21 / 10$ \\
BMI & $24.04 \pm 3.86$ & $25.40 \pm 4.43$ \\
Height & $164.85 \pm 6.72$ & $164.61 \pm 7.68$ \\
Weight & $65.55 \pm 12.38$ & $68.55 \pm 10.82$ \\
Smoking habit & $n=33$ & $n=31$ \\
Smoking & 29 & 21 \\
Non smoking & 4 & 10 \\
Course of disease & $n=33$ & $n=31$ \\
Course of disease (years) & $16.28 \pm 11.22$ & $17.60 \pm 14.91$ \\
Scale score & $n=33$ & $n=31$ \\
CAT & $16.30 \pm 5.80$ & $16.81 \pm 9.13$ \\
mMRC & $2.00 \pm 0.79$ & $2.23 \pm 1.06$ \\
Cough & $2.85 \pm 1.66$ & $2.13 \pm 1.71$ \\
Sputum & $2.18 \pm 1.04$ & $1.97 \pm 1.56$ \\
Wheezing & $2.73 \pm 1.31$ & $3.23 \pm 1.69$ \\
TCM symptoms & $12.97 \pm 3.85$ & $12.48 \pm 6.62$ \\
Spirometry & $n=33$ & $n=28$ \\
FEV1/FVC & $53.37 \pm 10.74$ & $50.87 \pm 11.95$ \\
FEV1 & $2.76 \pm 7.79$ & $1.14 \pm 0.57$ \\
\hline
\end{tabular}

TABLE 2: The change of CAT between the YQGB and $\mathrm{Pb}$ groups.

\begin{tabular}{|c|c|c|c|c|c|c|}
\hline & \multicolumn{2}{|c|}{$\begin{array}{l}\text { The change of } \\
\text { CAT after one } \\
\text { month }(n)\end{array}$} & \multicolumn{2}{|c|}{$\begin{array}{c}\text { The change of } \\
\text { CAT after two } \\
\text { months }(n)\end{array}$} & \multicolumn{2}{|c|}{$\begin{array}{l}\text { The change of } \\
\text { CAT after three } \\
\text { months }(n)\end{array}$} \\
\hline & $\begin{array}{c}\geq 2 \\
\text { points }\end{array}$ & $\begin{array}{c}<2 \\
\text { points }\end{array}$ & $\begin{array}{c}\geq 2 \\
\text { points }\end{array}$ & $\begin{array}{c}<2 \\
\text { points }\end{array}$ & $\begin{array}{c}\geq 2 \\
\text { points }\end{array}$ & $\begin{array}{c}<2 \\
\text { points }\end{array}$ \\
\hline YQGB & 27 & 5 & 32 & 1 & 33 & 0 \\
\hline $\mathrm{Pb}$ & 18 & 10 & 20 & 4 & 25 & 6 \\
\hline$X^{2}$ & \multicolumn{2}{|c|}{3.214} & \multicolumn{2}{|c|}{3.229} & \multicolumn{2}{|c|}{7.048} \\
\hline$P$ & \multicolumn{2}{|c|}{0.073} & \multicolumn{2}{|c|}{0.072} & \multicolumn{2}{|c|}{0.01} \\
\hline
\end{tabular}

3.2.3. Pulmonary Functions before and after the Treatment between the YQGB and $\mathrm{Pb}$ Groups. There were no statistical significant differences in these parameters at different time points compared to pretreatment values in both YQGB group and $\mathrm{Pb}$ group (Figures 3-8).

3.2.4. CAT, mMRC, Main Symptom, and TCM Syndrome Scores before and after the Treatment between the YQGB and $\mathrm{Pb}$ Groups. After one month, in the YQGB group CAT scores were lower than pretreatment values $(P<0.01)$; cough, sputum, and wheezing symptom scores were also lower $(P<$ $0.05)$, as well as TCM syndrome scores $(P<0.01)$. In the $\mathrm{Pb}$ group, CAT was lower than pretreatment values $(P<0.05)$ (Figures 9 and 10).

After two months, in the YQGB group, CAT scores were lower than pretreatment values $(P<0.01)$, as well as cough, sputum, and wheezing symptom scores $(P<0.01, P<0.01$, and $P<0.05)$, and TCM syndrome scores $(P<0.01)$. In the 

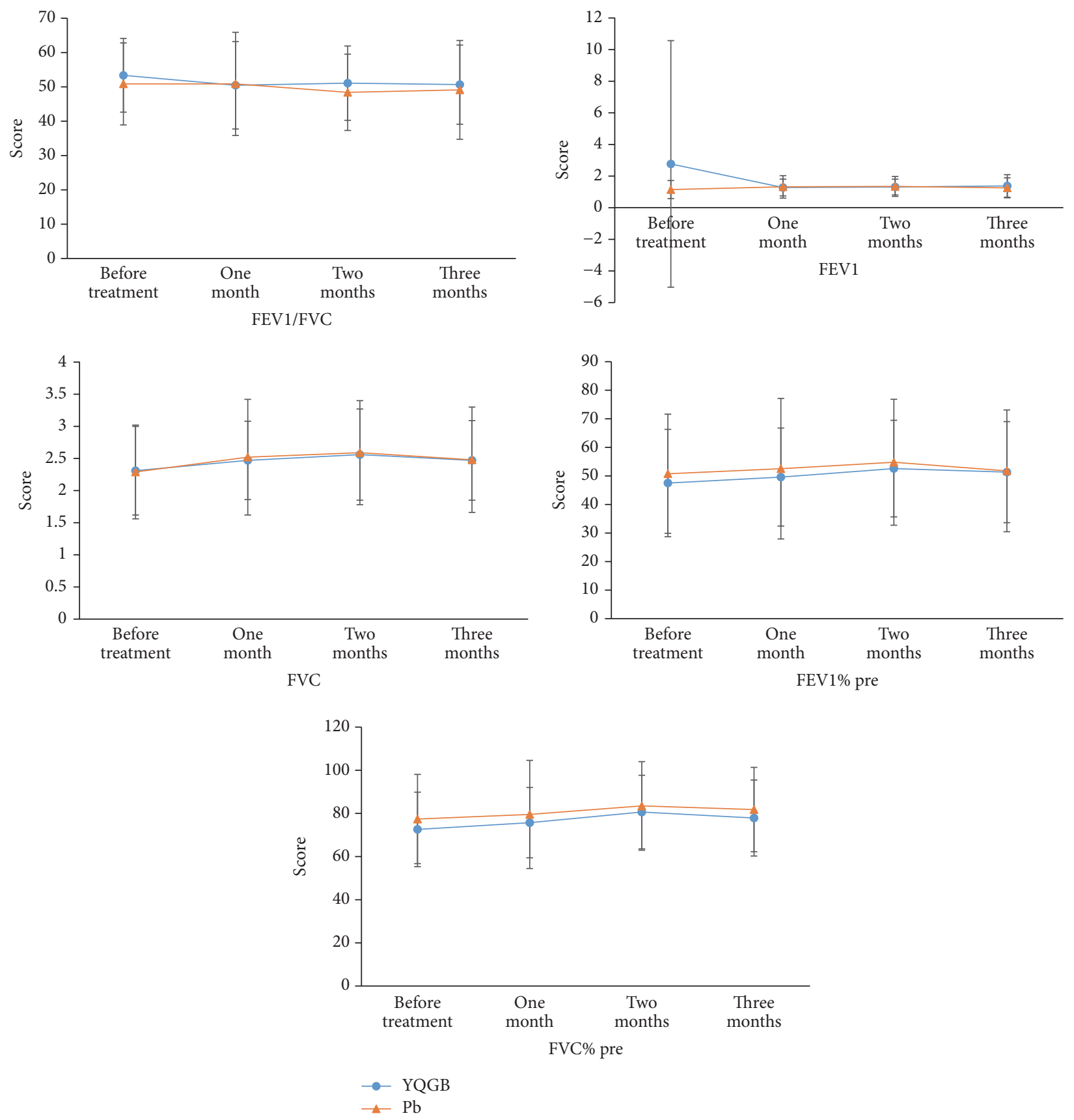

FIGURE 1: Pulmonary functions in the two groups in three months of treatment.

$\mathrm{Pb}$ group, only CAT and sputum symptom scores were lower than pretreatment values $(P<0.01, P<0.05)$ (Figures 11 and 12).

After three months, in the YQGB group, CAT scores were lower than pretreatment values $(P<0.01)$, as well as cough, sputum, and wheezing symptom scores $(P<0.01)$ and TCM syndrome scores $(P<0.01)$. In the $\mathrm{Pb}$ group, CAT scores were lower than pretreatment values $(P<0.01)$, as well as cough, sputum, and wheezing symptom scores $(P<0.05)$ and TCM syndrome scores $(P<0.01)$ (Figures 13 and 14$)$.
3.3. Adverse Reactions. There were no abnormal changes of clinical significance in laboratory indicators such as blood and urine routine tests, renal function, and ECG. Moreover, there were no adverse reactions such as nausea, abdominal pain, and diarrhea.

\section{Discussion}

COPD prevalence is $8.2 \%$ in the subpopulation above 40 years old in the seven provinces in China [11] and $13.4 \%$ in Korea [12]. COPD is an important cause of disability and 

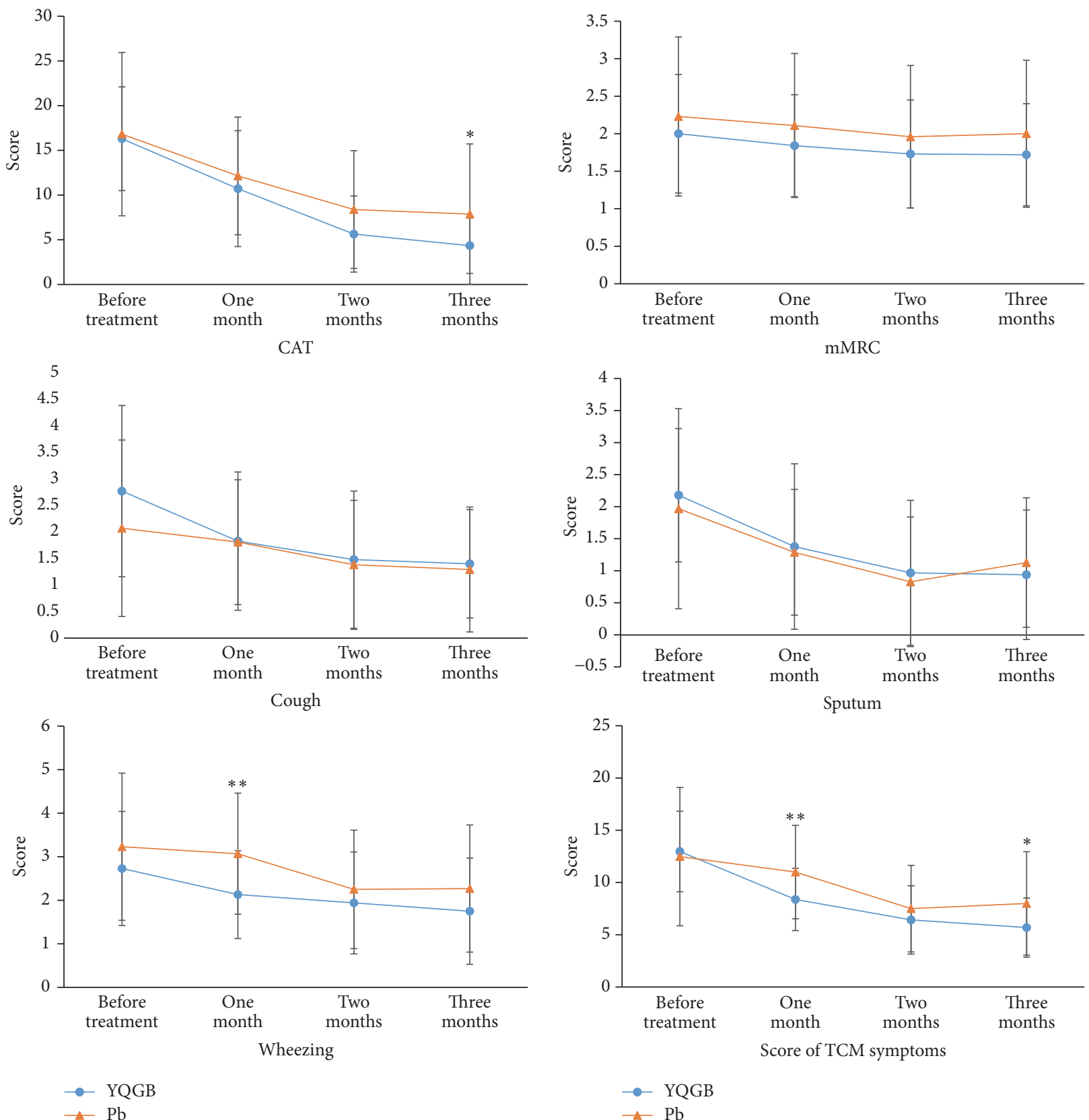

Figure 2: CAT, mMRC, and TCM syndrome scores in the two groups in three months of treatment. ${ }^{*} P<0.05,{ }^{* *} P<0.01$.

death worldwide, imposing huge and growing economic and social burden to mankind [13]. Therefore, how to effectively control COPD has become a major public health problem that needs urgent solution. COPD is a disorder of progressive airflow limitation caused by chronic inflammation of the airways and lung parenchyma; it is associated with symptoms such as cough, sputum production, and dyspnea [14]. However, the risk of acute exacerbation in COPD patients with symptoms of chronic cough and sputum is increased by 4.15 times, with the risk of hospitalization due to acute exacerbation increasing by 4.08 times [15]. Meanwhile, increasing symptom burden is associated with higher health care resource utilization with a detrimental impact on work productivity [16]. Therefore, symptomatic treatment has a great clinical appeal for COPD patients. However, treatment according to syndrome difference based on the four diagnostic principles of "diagnosis through observation, diagnosis through auscultation and olfaction, diagnosis through inquiry, and diagnosis through pulse feeling" is the characteristic and one of the advantages of traditional Chinese medicine.

The Yi Qi Gu Biao pill, a compound preparation including Chinese herbs (Salvia miltiorrhiza Bunge, Blighted wheat, Atractylodes macrocephala Koidz, Pinellia ternate, Citrus reticulata Blanco, Perilla frutescens (L.) Britt., Poria cocos (Schw.) Wolf, Saposhnikovia divaricata (Trucz.) Schischk., 


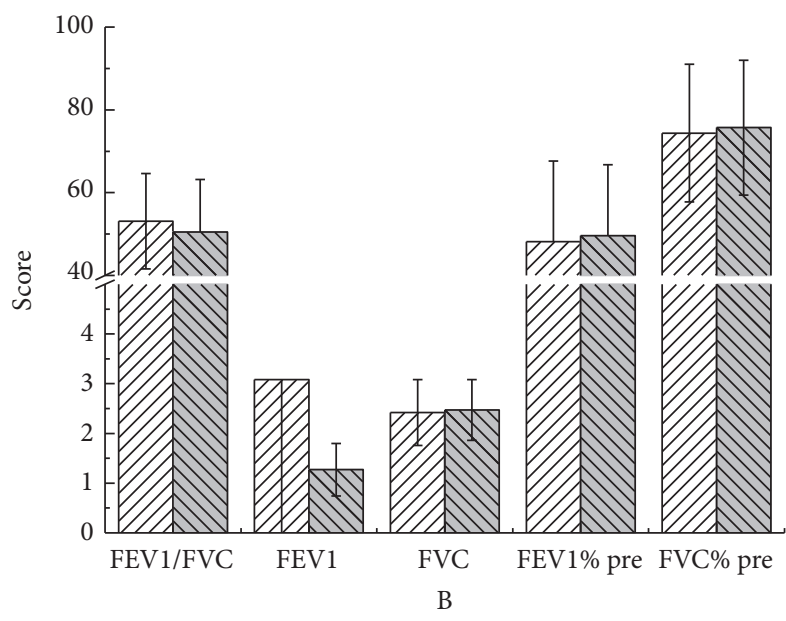

ZII Before the treatment

IIV After one month

FIGURE 3: Pulmonary functions in the YQGB group after one month and before treatment.

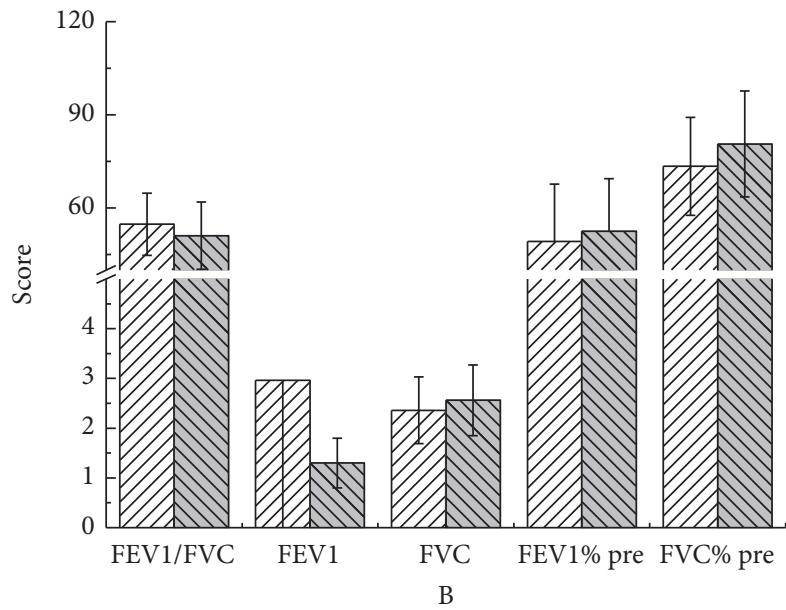

ZII Before the treatment III After two months

FIgURE 4: Pulmonary functions in the YQGB group after two months and before the treatment.

Coix lacryma-jobi L. war.ma-yuen (Roman.) Stapf, Tussilago farfara L., Scutellaria baicalensis Georgi, Fritillaria cirrhosa D. Don, and Eriobotrya japonica (Thunb.) Lindl.) is used to treat $\mathrm{COPD}$ at a stable stage. We found that the Yi Qi Gu Biao pill improves the quality of life of COPD patients at a stable stage, reducing the number of annual acute exacerbation events [5]. COPD is a publicly recognized heterogeneous disease, with therapeutic responses greatly differing even for patients with the same symptoms and drugs [17]. Based on a previous study, to evaluate the efficacy and safety of the Yi Qi Gu Biao pill for treating the frequent exacerbator phenotype in COPD (lung and spleen qi deficiency syndrome), we conducted a randomized, double-blind, placebo-controlled superiority trial. This study only included the exacerbator phenotype in COPD (lung and spleen qi deficiency syndrome), as subjects

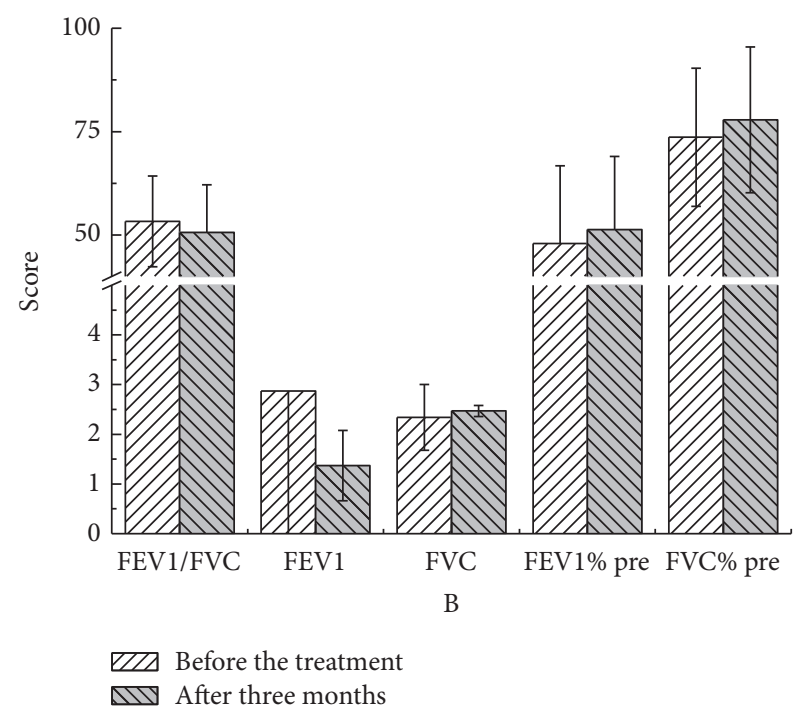

FIgURE 5: Pulmonary functions in the YQGB group after three months and before the treatment.

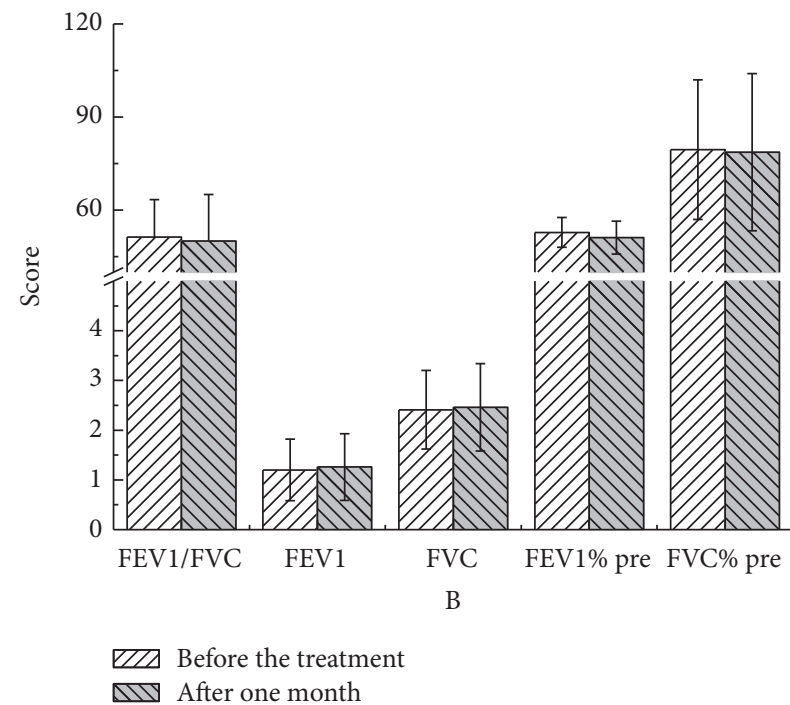

Figure 6: Pulmonary functions in the $\mathrm{Pb}$ group after one month and before the treatment.

to obtain a small sample size in order to standardize the group homogeneity of TCM syndrome differentiation and further clarify the effectiveness or ineffectiveness of the Yi Qi Gu Biao pill.

The Yi Qi Gu Biao pill had little impact on pulmonary function in patients with the exacerbator phenotype in COPD (lung and spleen qi deficiency syndrome), with equivalent efficacy with the $\mathrm{Pb}$ during the 3 months of treatment. However, FVC values after two and three months of treatment with Yi Qi Gu Biao pill were increased compared with pretreatment counterparts. Changes of pulmonary function parameters before and after the treatment were not significant. There were no statistically significant differences in the changes of pulmonary function parameters between the two groups after treatment with Yi Qi Gu Biao pill. 


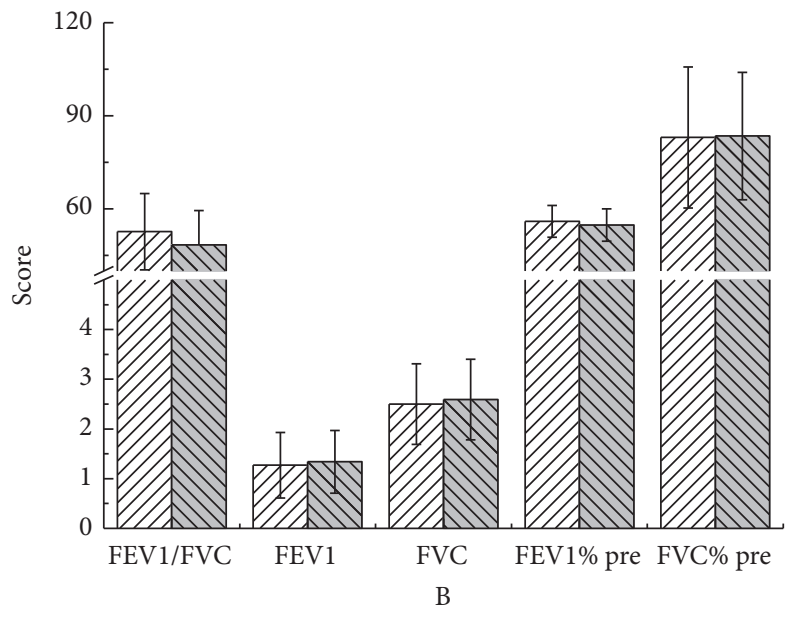

EII Before the treatment IIV After two months

Figure 7: Pulmonary functions in the Pb group after two months and before the treatment.

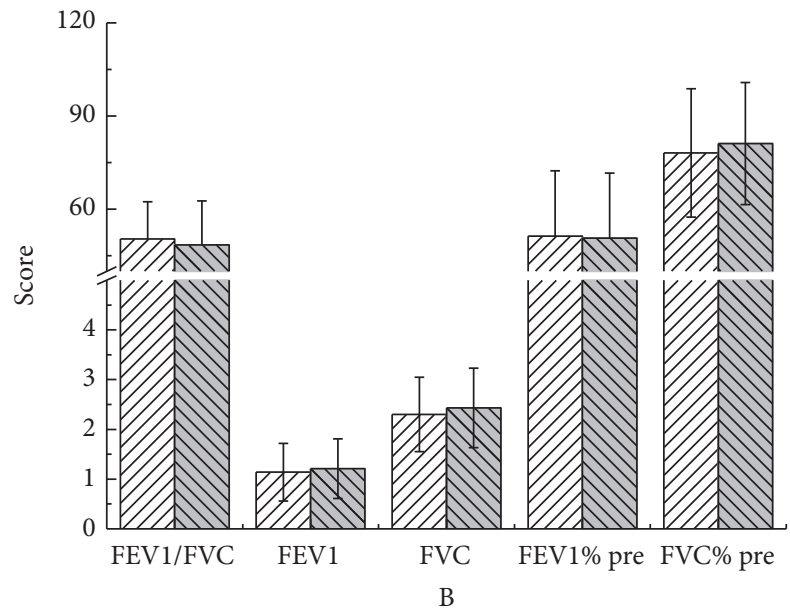

ZII Before the treatment NIV After three months

Figure 8: Pulmonary functions in the $\mathrm{Pb}$ group after three months and before the treatment.

Research showed that wheezing correlated with a higher possibility of acute exacerbation. Even among those who were treated according to the GOLD 2011 guidelines, patients with wheezing still had worse symptom scores and more exacerbations. Our study showed that wheezing symptom scores one month after treatment with Yi Qi Gu Biao pill were improved compared with those of the $\mathrm{Pb}$ group. Meanwhile, cough, sputum, and wheezing scores after one month of treatment with the Yi Qi Gu Biao pill were improved compared to pretreatment values. However, in the $\mathrm{Pb}$ group the cough, sputum, and wheezing scores did not improve until the third month suggesting that Yi Qi Gu Biao pill can cooperate with Western medicine to shorten the duration of cough, sputum, and wheezing symptoms in frequent exacerbator phenotype in COPD.

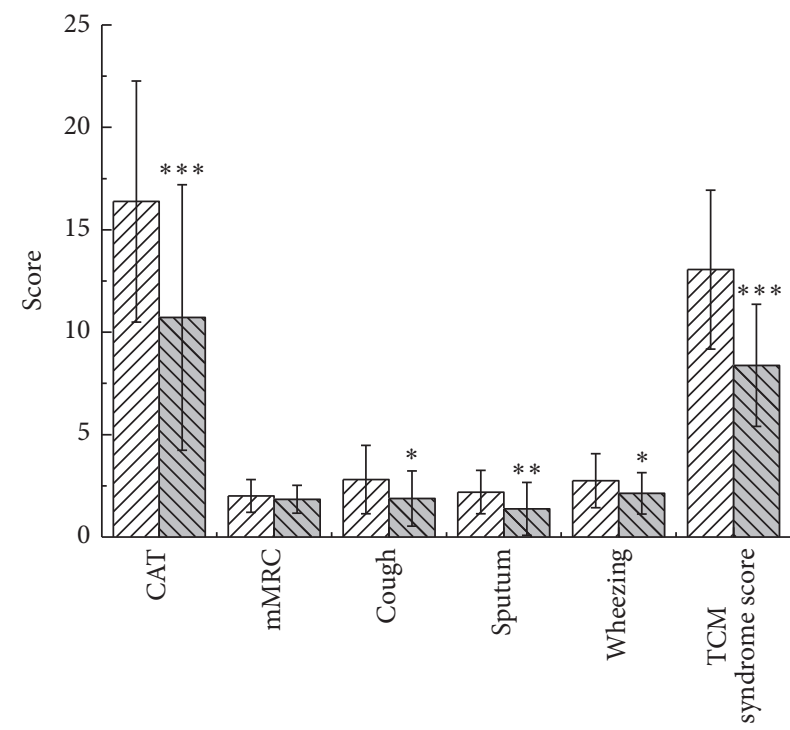

B

ZII Before the treatment MIV After one month

FIGURE 9: CAT and mMRC scores in the YQGB group after one month and before the treatment. ${ }^{*} P<0.05,{ }^{* *} P<0.01$, and ${ }^{* * *} P<0.001$.

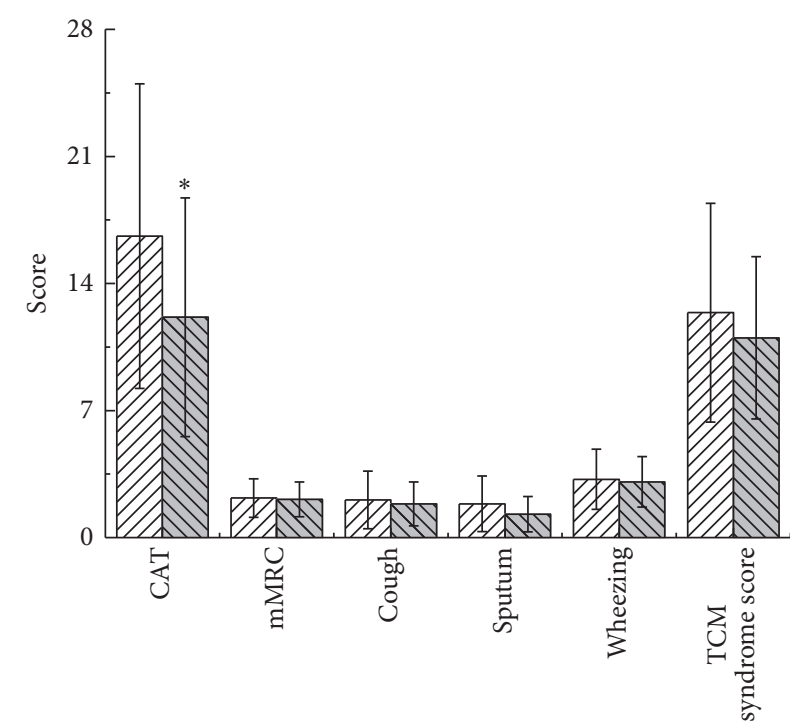

B

EII Before the treatment

MIV After one month

FIGURE 10: CAT and mMRC in the Pb group after one month and before the treatment. ${ }^{*} P<0.05$.

COPD assessment test (CAT) is a new health-related scale evaluating the health status of COPD patients [18], mainly for COPD patients at a stable stage [19]. After three months of treatment with the Yi Qi Gu Biao pill, CAT scores were reduced compared with the $\mathrm{Pb}$ group, indicating that prolonged and sustained administration of the drug is required for its effects of improving syndrome scores and 


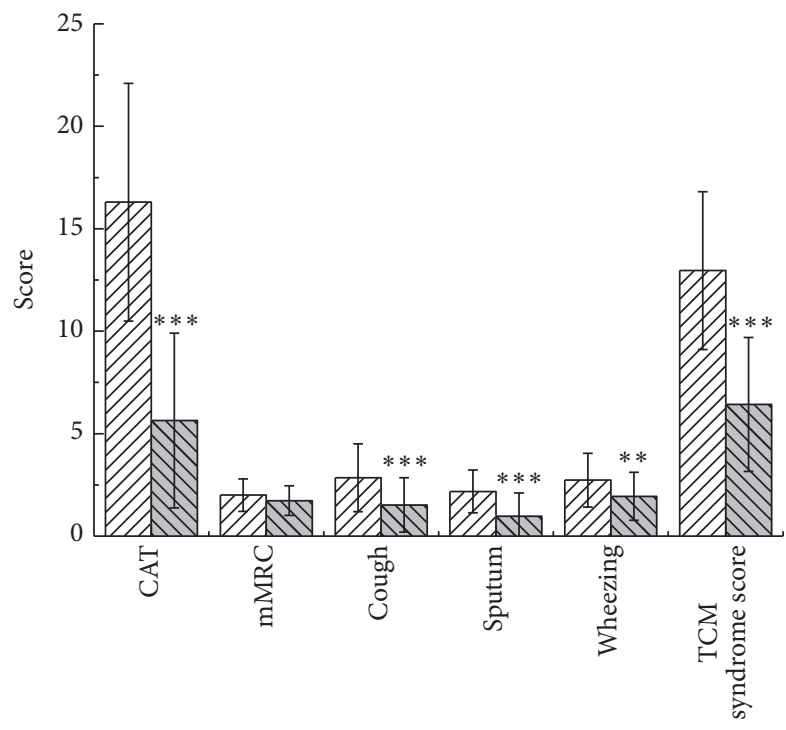

B

VID Before the treatment

NIV After two months

FIGURE 11: CAT and mMRC in the YQGB group after two months and before the treatment. ${ }^{* *} P<0.01,{ }^{* * *} P<0.001$.

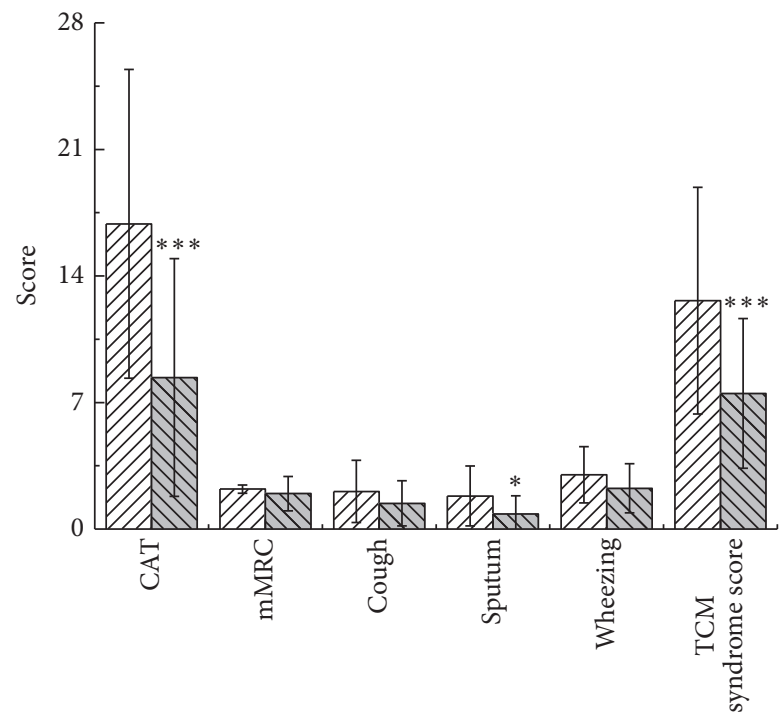

B

ZID Before the treatment MIV After two months

FIgURE 12: CAT and mMRC in the Pb group after two months and before the treatment. ${ }^{*} P<0.05,{ }^{* * *} P<0.001$.

the health status of patients ( $\geq 2$ months). Interestingly, Yi Qi Gu Biao pill could improve CAT scores after one month, as demonstrated by comparing pretreatment values with those after treatment. However, the degree of improvement varied, as reflected in differences between the two groups before and after the treatment; this means three months of treatment with the Yi Qi Gu Biao pill results in a higher

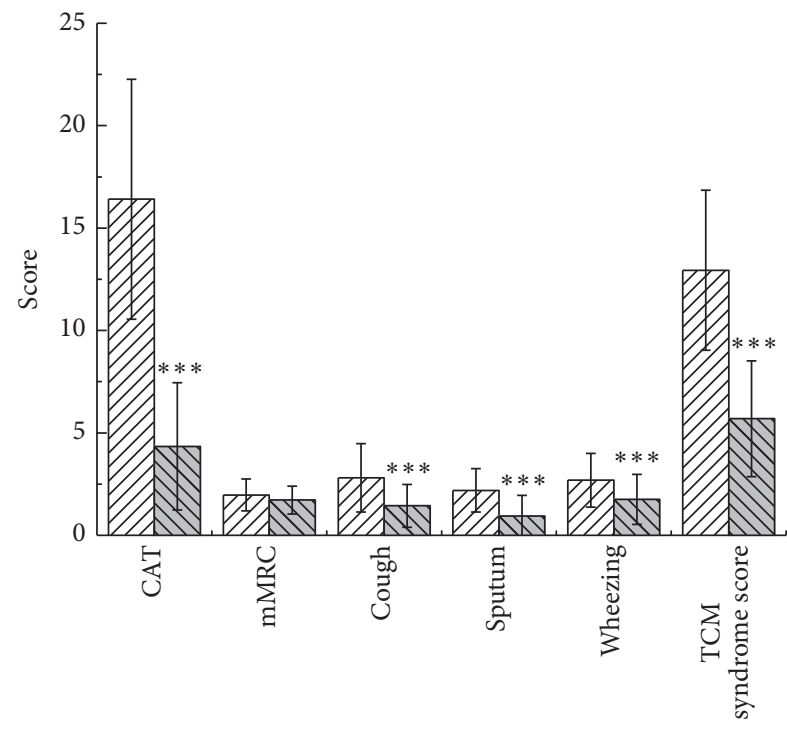

B

EID Before the treatment MIV After three months

FIGURE 13: CAT and mMRC in the YQGB group after three months and before the treatment. ${ }^{* * *} P<0.001$.

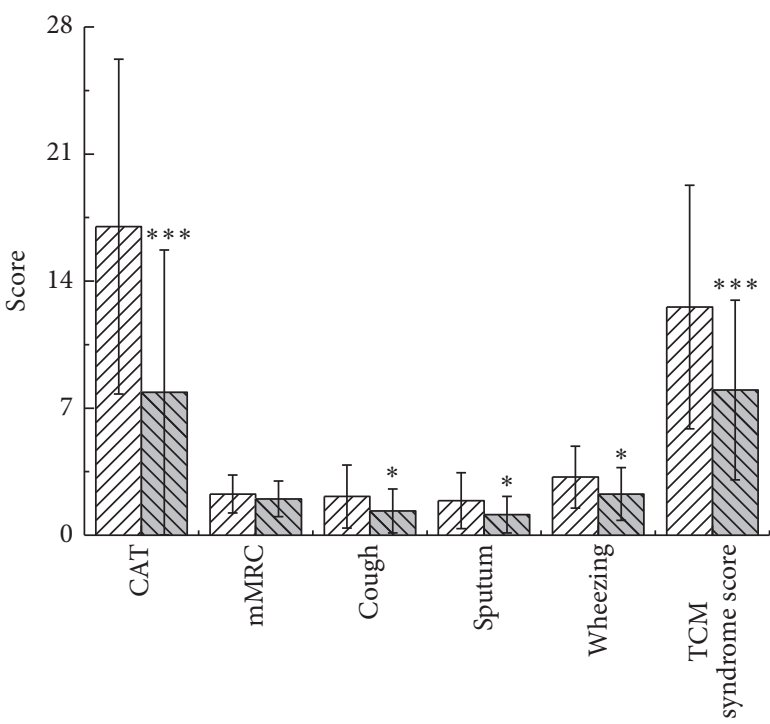

B

VID Before the treatment IIV After three months

FIgURE 14: CAT and mMRC in the $\mathrm{Pb}$ group after three months and before the treatment. ${ }^{*} P<0.05,{ }^{* * *} P<0.001$.

degree of improvement of CAT scores compared with $\mathrm{Pb}$ administration.

Improvement of TCM syndrome in the patients was better than $\mathrm{Pb}$ group at 1 and 3 months after treatment with the Yi Qi Gu Biao pill. Interestingly, TCM syndrome scores were improved compared with pretreatment values, after one month of treatment with the Yi Qi Gu Biao pill. However, 
such difference occurred in the $\mathrm{Pb}$ group only after two months of treatment.

Substantial placebo effect sizes have been documented for at least 50 years for various psychiatric symptoms [20]. Previous systematic reviews and meta-analyses have found placebo effect sizes that were inconsistent between studies and that have increased over time [21]. Findings [22] indicate that interactions between personality type and environmental cues may contribute to placebo responding. So, placebo effects, including patient's cognition, expectation, attention, preference, and communication with doctor as well as doctor's suggestion, expectation, and indirect regulation of diagnosis and treatment environment on patient's psychology, were essential factors for therapy efficacy. So, in this study, it showed improvements within the placebo groups.

\section{Conclusion}

The Yi Qi Gu Biao pill can improve wheezing, health status, and TCM syndrome in patients with frequent exacerbator phenotype in COPD (lung and spleen qi deficiency syndrome) and also can shorten the durations of cough, sputum, and wheezing.

\section{Ethical Approval}

This study was approved by the Ethics Committee of Traditional Chinese Medicine of Xinjiang Uygur Autonomous Region (Ethics Committee Document no. 2015XE0105).

\section{Conflicts of Interest}

The authors declare that they have no conflicts of interest.

\section{Acknowledgments}

This work is supported by National Natural Science Foundation of China (no. 81560728): Research of Plasma Proteomics in Patients with Frequent Exacerbator Phenotype in Chronic Obstructive Pulmonary Disease (Lung and Spleen Qi Deficiency Syndrome) Treated with Yi Qi Gu Biao Pill.

\section{References}

[1] M. K. Han, A. Agusti, P. M. Calverley et al., "Chronic obstructive pulmonary disease phenotypes: the future of COPD," American Journal of Respiratory and Critical Care Medicine, vol. 182, no. 5, pp. 598-604, 2010.

[2] M. Miravitlles, M. Calle, and J. J. Soler-Cataluna, "Clinical phenotypes of COPD: identification, definition and implications for guidelines," Archivos de Bronconeumología, vol. 48, no. 3, pp. 86-98, 2012.

[3] J. R. Hurst, J. Vestbo, A. Anzueto et al., "Susceptibility to exacerbation in chronic obstructive pulmonary disease," The New England Journal of Medicine, vol. 363, no. 12, pp. 1128-1138, 2010.

[4] J. A. Wedzicha, S. E. Brill, J. P. Allinson, and G. C. Donaldson, "Mechanisms and impact of the frequent exacerbator phenotype in chronic obstructive pulmonary disease," BMC Medicine, vol. 11, no. 1, article no. 181, 2013.
[5] F.-S. Li, Y.-L. Zhang, Z. Li et al., "Randomized, double-blind, placebo-controlled superiority trial of the Yiqigubiao pill for the treatment of patients with chronic obstructive pulmonary disease at a stable stage," Experimental and Therapeutic Medicine, vol. 12, no. 4, pp. 2477-2488, 2016.

[6] Z. Gao, U. Halmurat, and F. Li, "Study on the idea of frequent exacerbator phenotypeof chronic obstructive pulmonary disease (lung deficiency syndrome) in the joint diagnosis model of disease-phenotype-syndrome type," China Journal of Traditional Chinese Medicine and Pharmacy, vol. 31, no. 8, pp. 2905-2909, 2016.

[7] GOLD Executive Committee, "Global strategy for the diagnosis, management, and prevention of chronic obstructive pulmonary disease(COPD)," http://www.goldcopd.com/, 2011.

[8] Chinese Society of Traditional Chinese Medicine Branch of Pulmonary Disease Professional Committee, "Diagnosis criteria for TCM syndromes of chronic obstructive pulmonary disease (2011 edition)," Journal of Traditional Chinese Medicine, vol. 53, no. 2, pp. 177-178, 2012.

[9] J. Cai, T. Liu, and B. Cai, "Evaluation of clinical application significance of Chinese version of chronic obstructive pulmonary disease assessment test," Chinese Journal of Tuberculosis and Respiratory Diseases, vol. 34, no. 4, pp. 256-258, 2011.

[10] J. C. Bestall, E. A. Paul, R. Garrod, R. Garnham, P. W. Jones, and J. A. Wedzicha, "Usefulness of the Medical Research Council (MRC) dyspnoea scale as a measure of disability in patients with chronic obstructive pulmonary disease," Thorax, vol. 54, no. 7, pp. 581-586, 1999.

[11] N. Zhong, C. Wang, W. Yao et al., "Prevalence of chronic obstructive pulmonary disease in China: a large, populationbased survey," American Journal of Respiratory and Critical Care Medicine, vol. 176, no. 8, pp. 753-760, 2007.

[12] K. H. Yoo, Y. S. Kim, S. S. Sheen et al., "Prevalence of chronic obstructive pulmonary disease in korea: the fourth korean national health and nutrition examination survey, 2008," Respirology, vol. 16, no. 4, pp. 659-665, 2011.

[13] A. S. Buist, M. A. McBurnie, W. M. Vollmer et al., "International variation in the prevalence of COPD (The BOLD Study): a population-based prevalence study," The Lancet, vol. 370, no. 9589, pp. 741-750, 2007.

[14] H. M. Samaha, A. R. Elsaid, and E. NasrEldin, "Total serum IgE level in COPD patients," Egyptian Journal of Chest Diseases and Tuberculosis, vol. 64, no. 3, pp. 573-577, 2015.

[15] P.-R. Burgel, P. Nesme-Meyer, P. Chanez et al., "Cough and sputum production are associated with frequent exacerbations and hospitalizations in COPD subjects," CHEST, vol. 135, no. 4, pp. 975-982, 2009.

[16] B. Ding, M. Small, G. Bergström, and U. Holmgren, "COPD symptom burden: Impact on health care resource utilization, and work and activity impairment," International Journal of Chronic Obstructive Pulmonary Disease, vol. 12, pp. 677-689, 2017.

[17] C. Zhao and M. Zhou, "Research progress on the frequent exacerbator phenotype in chronic obstructive pulmonary disease," Chinese Journal of Tuberculosis and Respiratory Diseases, vol. 39, no. 12, pp. 973-975, 2016.

[18] P. W. Jones, G. Harding, P. Berry, I. Wiklund, W.-H. Chen, and N. K. Leidy, "Development and first validation of the COPD Assessment Test," European Respiratory Journal, vol. 34, no. 3, pp. 648-654, 2009.

[19] J. Vestbo, S. S. Hurd, A. G. Agustí et al., "Global strategy for the diagnosis, management, and prevention of chronic 
obstructive pulmonary disease: GOLD executive summary," American Journal of Respiratory and Critical Care Medicine, vol. 187, no. 4, pp. 347-365, 2013.

[20] K. Weimer, L. Colloca, and P. Enck, "Placebo effects in psychiatry: Mediators and moderators," The Lancet Psychiatry, vol. 2, no. 3, pp. 246-257, 2015.

[21] A. J. Hyde, B. H. May, C. C. Xue, and A. L. Zhang, "Variation in Placebo Effect Sizes in Clinical Trials of Oral Interventions for Management of the Behavioral and Psychological Symptoms of Dementia (BPSD): A Systematic Review and Meta-Analysis," The American Journal of Geriatric Psychiatry, 2016.

[22] M. Darragh, R. J. Booth, and N. S. Consedine, “'Oxytocin' for the outwardly oriented: Evidence for interactive effects in placebo responding," Journal of Psychosomatic Research, vol. 83, pp. 1015, 2016. 


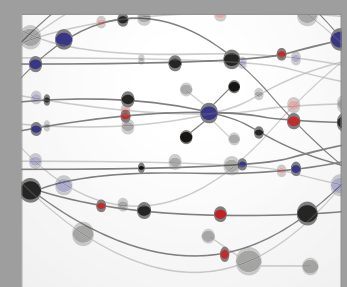

The Scientific World Journal
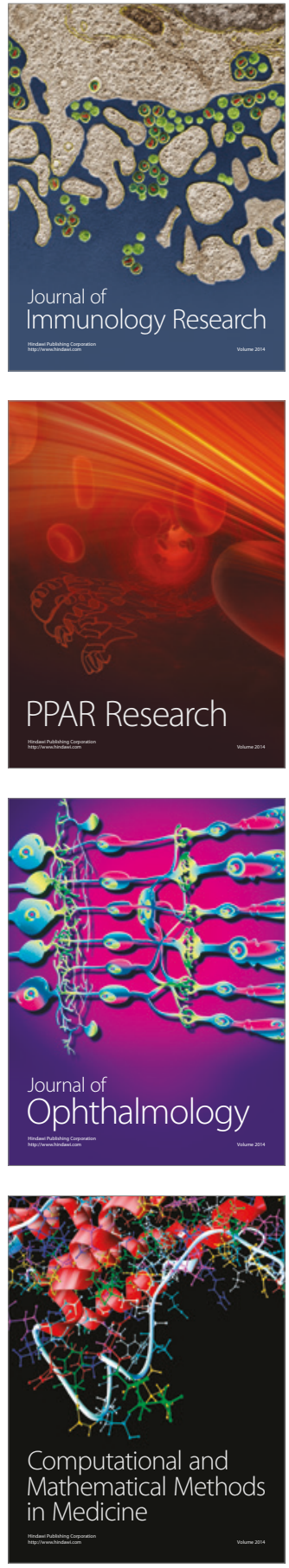

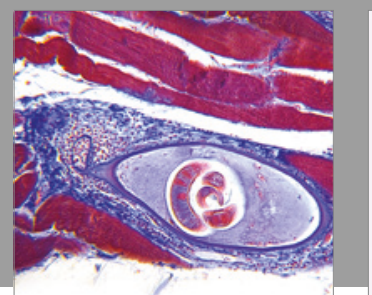

Gastroenterology Research and Practice
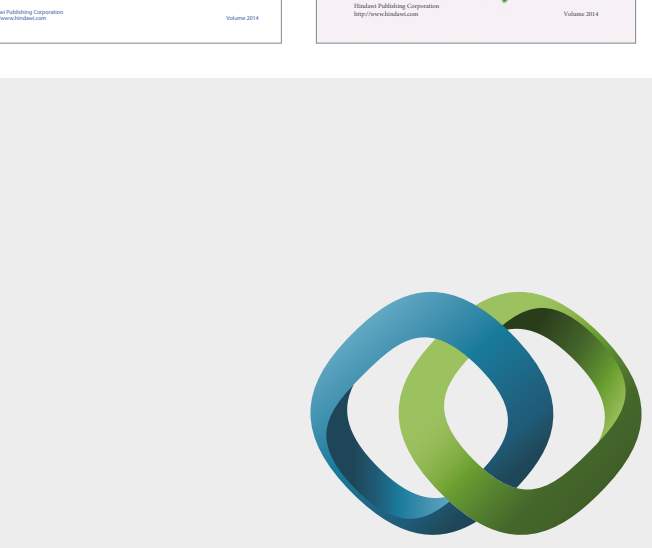

\section{Hindawi}

Submit your manuscripts at

https://www.hindawi.com
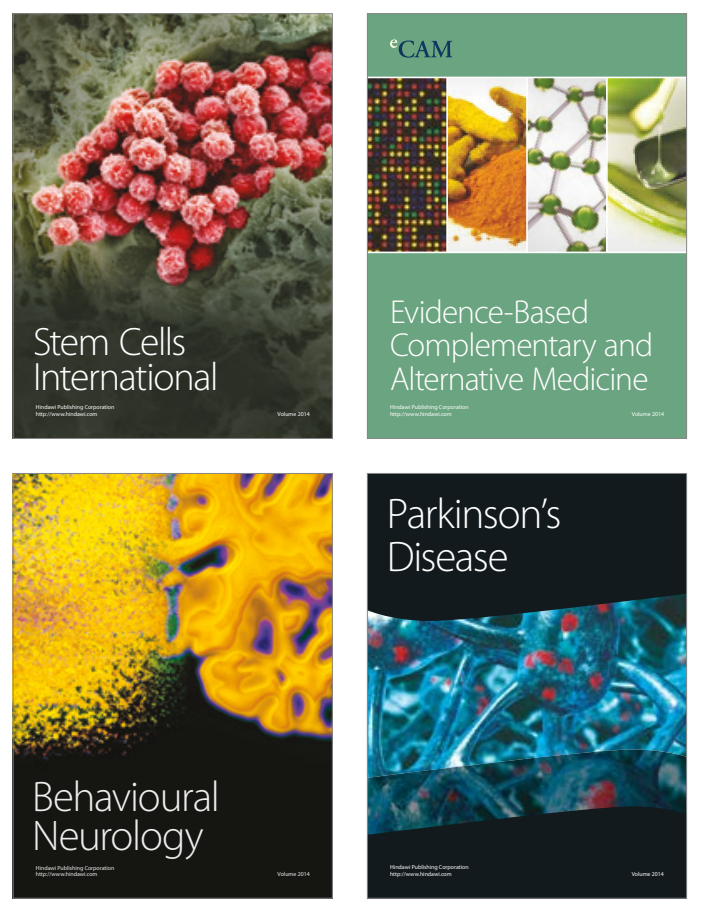
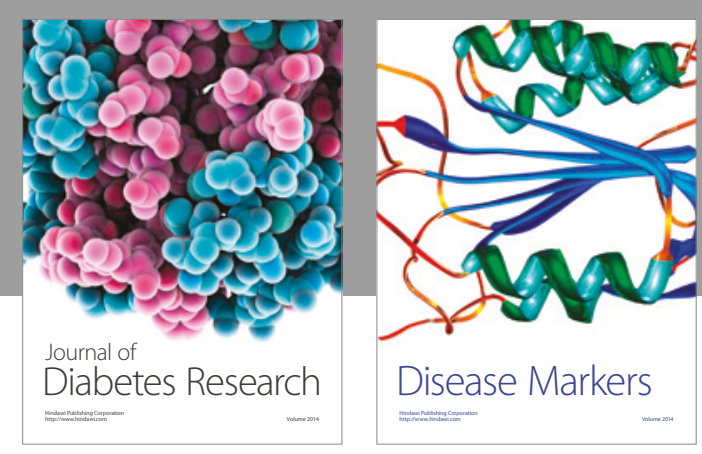

Disease Markers
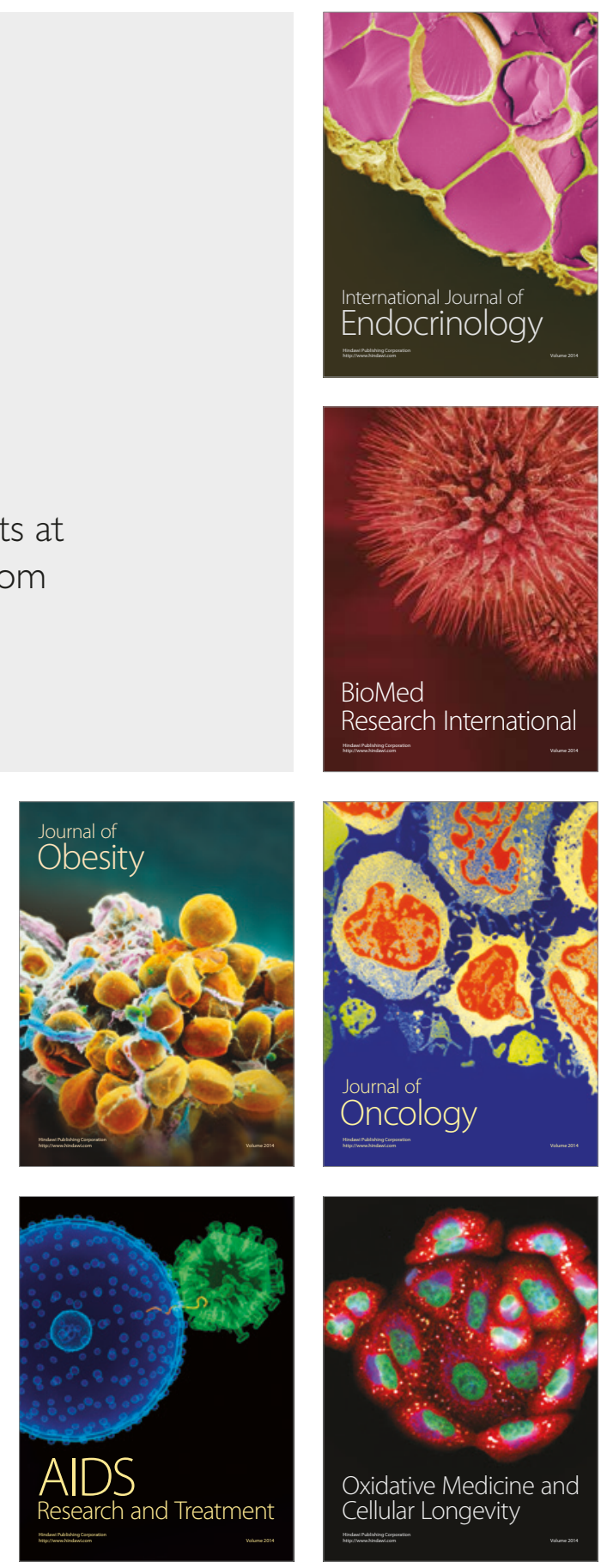